to improving training at all levels, rather than as a signal for introducing defensive practice. This book needs to be discussed by those who select medical students or recruit doctors and by all concerned with the education and welfare of doctors, and especially doctors in training. The importance of communication is a common theme throughout the book, and yet so often communication is considered as separate from clinical ability and competence. Those who are devising both the new curricula for doctors in postgraduate training, in order to encompass proposals of the Calman committee, and the content of continuing medical education need to act on the implications of the research described in this book.

Medical Accidents has set the academic background to the study of medical accidents; it is now the responsibility of the healthcare professionals to define and implement the practical applications. The quality of patient care can only benefit from such action.

Quality and Excellence in Human Services. Dickens P (pp 200, £14.95). Chichester: Wiley, 1994. ISBN 0471940542.

The British healthcare system is an unusual service industry. Its products are hard to define and market forces, when they apply at all, may be perverse. Workers who increase productivity are as likely to be penalised for "over performing" as to be rewarded. Purchasing power does not lie with the consumers but with purchasing authorities who, being accountable financially to a central body (the government), are often poor proxies. Most customers when dissatisfied with what they are being offered have no option other than to "take it or leave it."

Services are managed through rigid hierarchical structures with targets imposed from above (Health of the Nation) in a way reminiscent of the five year plans of Stalinist Russia. At the same time the great majority of available resources are committed by a group of workers - namely doctors - who not only do not account to the service managers for their professional decisions but are often in an adversarial relationship to them. These workers are not trained to view the recipients of the service as consumers, and they view quality more as "doing the right thing" than as "satisfying the customers."

While acknowledging these issues, Paul Dickens argues that healthcare organisations are service industries and that industrial models of quality are relevant to them. As more and more providers and purchasers of health care seem to agree with him, and are attempting to introduce such models of quality and excellence, his book is timely.

Although it touches on "home grown" products such as clinical audit, the bulk of the book consists of a clear, readable, and critical account of the various approaches to quality used in industry. Brief descriptions of the work of four "quality gurus" are accompanied by relatively jargon free accounts of the principles behind their philosophies and the techniques and tools they developed. Thus the reader is given an introduction to quality costing, quality circles, and to some of the statistical methods employed in quality control procedures. The British standard on quality systems - BS5750/ IS09000 - is described and the suggestion made that more healthcare providers might seek accreditation.

The author seems to advocate introducing a modified form of total quality management into the healthcare services. For this to be more than just a time consuming paper exercise clinicians, and in particular doctors, would have to learn to work more as part of a team and pay much greater attention to patient satisfaction.

Possibly, however, there may be an incentive, that may also be a prerequisite, that will encourage clinicians to embrace total quality management. That is the promise it holds of catalysing a culture change within health service management. Total quality management must involve all within a healthcare organisation, including managers and purchasers. Each person must strive to improve the service he or she provides for customers. For clinicians the patient is the customer, for managers and support services the clinician is also a customer. Such a model puts a different complexion on the relationship between the clinician and human resource, finance, and medical records departments and may lead to a service that enhances the quality of clinicians' working lives.

PAUL LELLIOT Deputy Director,
Royal College of Psychiatrists

Computers and Clinical Audit: An Introduction for Doctors and Health Care Professionals. Kinn S, Siann T. (pp 200, £15.95 pb). Chapman and Hall, 1993. ISBN 0412492806.

The health service has been fascinated by computers since the $1960 \mathrm{~s}$. It seems axiomatic that such a data rich service would benefit from the most advanced forms of information handling. However, disaster has struck again and again, from the abandoned personal computer in the consultant's office to the large scale disasters such as those in the Wessex and West Midlands regions. As the publication of this book shows the introduction of audit has helped rekindle the fascination.

One major problem is communication. Professionals always invent their own languages to distinguish themselves from the common herd. Clinicians label a runny nose with the term rhinitis, the systems analyst labels the start up procedure for a computer -AUTO EXEC.BAT. Thus incomprehension grows as each professional spends years learning his or her own language. The first nine chapters of the book are an excellent basic primer for the first lessons in computer language; only the final three discuss the application to audit. A language is best learnt by use - be it French or "computerese." The earlier in life one learns a language the easier. As computer studies are now part of the school curriculum, hopefully the gap will close. However, as the computer is only a tool in audit or elsewhere consumer demand will accelerate the trend to simpler interfaces, and, hopefully, the book's next edition will have only three chapters or fewer as a basic primer!

The last three chapters emphasise that data collection with the use of computers is involved in only the second (measuring current performance) of the four stages of the audit cycle. Equal priority must be given to setting standards, assessing performance against standards, and identifying the need for and implementing change. The audit must be planned first and then the use of a computer considered. However, for large audit projects the use of a computer becomes essential and the value of integrating good data through large scale computer systems is self evident. The increasing cost in equipment, training, and planning leads to many pitfalls, which in the book are clearly laid out.

The ultimate goal for audit is for it to be embedded in both the ethos and practice of the health service. The tool to enable this is the computer. The ultimate goal for the computer is for it to be integrated into the daily work of the hospital, probably through a hospital information support system. Every user of that system is supported in their job. Their data are integrated to produce information on which to base audit, thus reducing the extra data required for each individual audit.

CHARLES PANTIN Consultant Physician

Review of Seven Mental Health Information Systems: A Functional Perspective. Lelliott P, Flannigan C, Shanks S (39 pp, £5). London: Royal College of Psychiatrists, 1993. ISBN 0902241591 .

This first publication from the research unit of the Royal College of Psychiatrists reflects a commitment to share the experience of projects undertaken by the unit with a wider professional audience. Here are brief descriptions of seven systems, all developed with the active involvement of mental health practitioners. Each was demonstrated to the college's visiting panel and the capabilities of the seven systems were compared against a standard set of questions that captured the essential needs of mental health information systems.

The rate of change in the structure of the NHS and in its information requirements is apparent from the descriptions of the psychiatric services in which the systems are working. There is hardly a mention of the application to contract information requirements, and most of the systems described were unable to link with the patient administration system. Some of the systems have the potential of helping with clinical audit and quality programmes, but little attention has so far been given to these applications. Disappointingly, most of the systems are not being used directly by clinicians. It would have been helpful if the review had given some suggestions about the most effective approaches in gaining clinician ownership. 
The two most valuable features of this review are, firstly, its largely non-technical summary of each scheme which gives a "warts and all" picture (the authors do not give a "best buy" since the limitations of each system are spelt out), and, secondly, the commonsense and clinically relevant checklist of requirements and expectations which the investigators used.

Four potential clinical applications were tested: all the information systems were able to identify current inpatients and their Mental Health Act status, but only three systems - Plymouth (MCHII), Ealing (Psy-Mon), and Hackney (MHL) covered patients in contact with community mental health centres. More of the systems were able to summarise past psychiatric contacts for patients, but only inpatient contacts were consistently covered. None of the systems could produce a complete care plan linking problems at admission with problems at discharge - and only three of the systems recorded clinical ratings that could be used for outcome measurements: Huddersfield (CIS), Ealing (Psy-Mon), and West Hampstead (Safety Net).

The review has been popular among psychiatrists, largely because it is a useful and readable introduction to the application of information systems to mental health, but it will add little to the knowledge of most clinical audit staff and those who have already undertaken serious work in this area. The time gap from the initial visits means that it cannot be relied on to give current capabilities of the seven systems described.

PEITIR JIIIIEERY Clinical Audit I cal.

Harrose and Hillingdon Healthare $\backslash$ HS Tims

\section{MEETINGS REPORT}

Ninth family practitioners' congress,
Cape Town, South Africa, April 1994

The extensive media reports in the United Kingdom about events in South Africa leading up to the first democratic election hardly mentioned health care. However, the health system in South Africa reflects the political and economic divisions that are a legacy of apartheid. At one extreme, a small section of the population has access to high quality medicine as technologically advanced as that anywhere in the world, while at the other, large numbers of the population have virtually no access to health care at all. The commonest cause of death is gastroenteritis. The enormous task of reorganising health care to meet the needs of the entire population was the principal theme of the ninth family practitioners' congress held in Cape Town a few days before the election. Outside the conference hall the streets were decorated with posters of the African National Congress and National Party, but there were no signs of tension or reports of political violence in the area, in contrast to the impressions conveyed by news reports.

In the opening address, Dr F Van $\mathrm{Zy}$ Slabbert, once a leading member of the Democratic Party stated his view that the elections and the immediate post election period would be fairly quiet but that major problems could arise within two to three vears if the majority of the population does not see that reforms have taken place and the quality of their lives has improved. Providing primary care for all is therefore a key issue. Family practitioners at the congress were united in agreeing that they could do much towards improving equity and quality of primary care. Highly critical of the emphasis on tertiary care they had encountered in medical schools, they claimed that $50 \%$ of medical students from one medical school, once graduated, left the country. Certainly, there was vocal agreement that the medical education system was failing to provide the kind of primary care doctor that South Africa requires.

Some of the presentations told of heroic efforts to revolutionise primary health care. Some doctors had helped set up small scale industries in deprived communities and undertaken basic town planning in addition to the more traditional activities of family practice. Others were studying the potential role of nurse practitioners as a source of inexpensive primary care for all. Would this be an effective option or would nurse practitioners be a challenge to the long term role of family doctors? Also some doctors were anxious that in the future South Africa they would no longer be able to provide the kind of service that affluent whites had come to expect, such as long consultations and the support of modern technology. Would changes to the health service lead to a reduction in its quality for some?

Reflecting many of these issues, the presentations ranged from the content of undergraduate training and education, family therapy, and quality assurance to different approaches to meeting the needs of the deprived majority. On the same platform presentations that would be unremarkable in a programme in western Europe were found in partnership with discussion of the basic necessities for health, such as a clean water supply and immunisation. In one set of presentations, a paper on chronic under-nutrition was followed by one on overweight and obesity management. The problems are enormous and difficult to grasp, but there is general good will and support for progress. Some individuals are working tirelessly to revolutionise health care.
Perhaps in ten or twenty years' time they will be considered as heroes in the mould of Florence Nightingale. RICHARD BAKER
Dirctor, lili Lilly. National Climical Audit Contr.

\section{DIARY}

\section{2-24 February 1994}

Bradford: Bankfield Hotel, Bingley.

Third national MAAG conference. The evolution of medical audit. ( $£, 350$ approximately including accommodation.) Further details from MAAG Office, Bradford Family Health Services Authority, Joseph Brennan House, Sunbridge Road, Bradford BD1 2SY (tel 0274 724575 ext 212; fax 0274394245).

\section{May - 2 June}

St John's, Newfoundland: Twelfth World Congress of the International Society for Quality Assurance (ISQA). Partnerships for creating a quality health system. Second announcement. Further details from the conference conductor, 1995 ISQA Organising Secretariat, Beclin Building, 1118 Topsail Road, PO Box 8234, St John's, Newfoundland, Canada A1B 3N4 (tel (709) 3647704 ; fax (709) $3646460)$.

\section{NOTES}

The 1995 Baxter Award for Healthcare Management in Europe, valued at US $\$ 5000$, will be presented by Tho European Healthcare Management Association EHMA), either to an individual or to an organisation, for an outstanding publication or practical contribution to excellence in healthcare management in Europe, or both. Contributions will be considered in any of the following: management development initiatives, innovations in management practice, and health services research. A crucial test will be practical impact (either proven or potential) on healthcare management in Europe. The award is open to anvone associated with the health care sector. Closing date for receipt of submissions is 28 February 1995. For guidelines on submissions contact Rena Dooley, Manager Membership Services, European Healthcare Management Association, Vergemount Hall, Clonskeagh, Dublin 6, Ireland. (tel +353.1.283 9299; fax $+353.1 .2838653)$. 